Please do not remove this page

RMIT

UNIVERSITY

\title{
A blind equalization algorithm for wireless multimedia communication
}

Jusak, Jusak; Athanasiadis, Tasso; Hussain, Zahir

https://researchrepository.rmit.edu.au/esploro/outputs/9921858807801341/filesAndLinks?institution=61RMIT_INST\&index=null

Jusak, J., Athanasiadis, T., \& Hussain, Z. (2005). A blind equalization algorithm for wireless multimedia communication. Proceedings of the 2005 Asia-Pacific Conference on Communications, 931-935.

https://doi.org/10.1109/APCC.2005.1554199

Published Version: https://doi.org/10.1109/APCC.2005.1554199

Repository homepage: https://researchrepository.rmit.edu.au

(c) 2005 IEEE. Personal use of this material is permitted. However, permission to reprint/republish this material for advertising or promotional purposes or for creating new collective works for resale or redistribution to servers or lists, or to reuse any copyrighted component of this work in other works must be obtained from the IEEE.

Downloaded On 2023/04/27 00:25:12 +1000 


\title{
A Blind Equalization Algorithm for Wireless Multimedia Communication
}

\author{
Jusak Jusak, Student Member, IEEE, Tasso Athanasiadis, and Zahir M. Hussain, Senior Member, IEEE \\ School of Electrical and Computer Engineering \\ RMIT University, Melbourne, Victoria 3000, Australia \\ s3001898@student.rmit.edu.au, s8902991@student.rmit.edu.au,zmhussain@ieee.org
}

\begin{abstract}
In the last decade much research effort has been dedicated to deal with the issues of wireless multimedia communication, in particular, the bandwidth limitation (capacity) and channel impairment (intersymbol interference - ISI) experienced by wireless communication systems in the transmission of multimedia data such as, digital images/video. To overcome the problem of ISI caused by multipath propagation in a wireless environment, various channel coding and equalization schemes have been developed. We have recently proposed a new scheme for blind channel equalization. In this paper, we test this scheme for wireless image transmission. Simulation results show that the low complexity of implementation and the fast convergence rate are the major advantages of employing the new scheme for multimedia applications. It is also shown, from perceptual-based analysis as well as objective measurements using peak signal-tonoise ratio (PSNR) of the recovered image, that the recentlyproposed blind adaptive equalization algorithm outperforms existing methods.
\end{abstract}

\section{INTRODUCTION}

Over the past decade, the demand for multimedia applications and services over wireless networks such as $3 \mathrm{G}$ mobile communication systems has grown considerably. Reported studies into next-generation (NG) wireless/ mobile networks [1] have shown a significant trend towards personalized mobile communications - ubiquitous computing - and a stronger need for wireless multimedia-equipped devices. This has stimulated a significant increase in the amount of wireless data transmitted due to multimedia content, causing a major shift from traditional wired telephony-oriented services, which have predominantly supported the transmission of voice data.

The processing of digital video, images, and audio signals using sophisticated and quite complex algorithms (codecs) are at the heart of many modern communication products, from personal digital assistants (PDAs) to video-capable cell phones. Understanding the characteristics of wireless digital communication systems designed for the transmission of multimedia signals is essential for developers of the systems that target multimedia applications.

One of the main issues with mobile multimedia communication, however, is the degradation in both communication channel performance, system latency, and acceptable quality of multimedia data received on wireless terminals/ devices. This is mainly due to the inherent characteristics of wireless communication systems - the time-varying nature of wireless channel conditions and propagation environments [2].

Despite the ongoing development of key communication technologies to deal with multimedia applications, such as adaptive compression [3] and adaptive modulation and coding
[4], the transmission of multimedia data over wireless communication channels still introduces significant bottlenecks. This is mainly due to the fact that representing multimedia data such as digital audio/ images/ video requires a large amount of information, leading to high bandwidth, computation energy, and communication energy requirements.

The communication bandwidth available to wireless/mobile systems for the transmission of multimedia data, and the processing capabilities of the communication system/devices, are often severely limited. Mobile radio channels must, therefore, transmit user information in a highly compressed form, while making efficient use of available frequency spectrum and communication power.

As a result, new ways of 'adaptively' compressing, modulating, encoding and transmitting multimedia data (by dynamically controlling communication parameters to suit variations in channel conditions, computational requirements, and available processing resources) have emerged [5], [6], [7], [8]. Furthermore, due to the demand for high data rate transmission of multimedia signals over video-capable wireless networks, channel equalization has become essential in dealing with the problems associated with intersymbol interference (ISI). On the other hand, space-time coding (STC) and orthogonal frequency division multiplexing (OFDM) have emerged as powerful techniques to minimize the system bit error rate, and hence opened the way towards increasing data rates to support multimedia applications as we have shown in [9].

Blind equalization has been extensively used in communication systems to remove the ISI produced by dispersive channels [10], and has become increasingly important where full bandwidth utilization of the channel is necessary. It is well known that conventional equalization techniques rely on the transmission of training signals which leads to a reduction in channel bandwidth and allocated resources. The main advantage of using a 'blind' system is apparent where the use of training signals is both unrealistic and costly to implement.

The Constant Modulus Algorithm (CMA) that was originally proposed in [11], has attracted the main research effort as a suitable blind wireless channel equalizer - due to its robustness over the violation of perfect blind equalization (PBE) conditions [12]. To achieve the low complexity requirement of the CMA algorithm, recent studies associated with the fractionally-spaced equalization constant modulus (FSE$\mathrm{CM}$ ) algorithm have lead to the development of the dithered signed-error constant modulus algorithm (DSE-CMA) [13]. In this scheme, the input signal to the equalizer is dithered by 


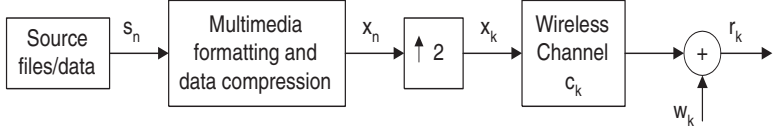

Fig. 1. Transmitter block for $T / 2$ baseband communication system.

a non-subtractive sinusoidally-distributed signal before a sign operation is applied.

In this paper, we investigate the characteristics and system performance of a wireless multimedia communication system using the DSE-CMA blind equalization technique in the application of compressed images. The framework developed can be extended to study the transmission of other types of multimedia signals such as, high rate digital video. Empirical results obtained are used to determine subjective (perceptualbased) quality issues, without the need for an extensive numerical analysis; as is often required in most research studies.

\section{System Model}

Fig. 1 and Fig. 2 depict a multirate model of a baseband communication system comprising of a transmitter and receiver part, where subscript $n$ denotes baud-spaced quantities and subscript $k$ represents $T / 2$ fractionally-spaced quantities. Through this system, a sequence of formatted multimedia data symbolized as $x_{n}$ with $T$ symbol period is transmitted through the $T / 2$-spaced channel c of a length $N_{c}$. In many communication systems, even in the absence of noise the effects of filtering and channel-induced distortion lead to intersymbol interference (ISI). Therefore, in addition to the white Gaussian noise the received signal is also corrupted by ISI. These distorted signals can only be removed by employing an equalizer in the receiver. Following the figures, the system output of the equalization may be expressed as:

$$
\begin{aligned}
y_{n} & =\mathbf{r}^{H}(n) \mathbf{f}(n)+\mathbf{w}^{H}(n) \mathbf{f}(n) \\
& =\mathbf{x}^{H}(n) \mathbf{C} \mathbf{f}(n)+\mathbf{w}^{H}(n) \mathbf{f}(n),
\end{aligned}
$$

where $x(n)=\left[x_{n}, x_{n-1}, \cdots, x_{n-N_{x}+1}\right]^{t}$ denotes a finitelength source symbol vector of a length $N_{x}=\left\lfloor\left(N_{c}+\right.\right.$ $\left.\left.N_{f}-1\right) / 2\right\rfloor$, while $\mathbf{f}$ is representing a column vector of fractionally-spaced equalizer coefficients with a length $N_{f}$. A column vector $\mathbf{r}(n)$ and $\mathbf{w}(n)$ be the symbol of timedecimated of $N_{f}$ received samples and white Gaussian noise, respectively. A Hermitian operator is denoted by $(.)^{H}$, and matrix transposition is symbolized by $(.)^{t}$. The matrix $\mathbf{C}$ symbolizes a $N_{x} \times N_{f}$ time-decimated channel convolution, defined as follows:

$$
\left[\begin{array}{ccccccc}
c_{1} & c_{0} & & & & & \\
\vdots & \vdots & c_{1} & c_{0} & & & \\
c_{N_{c}-1} & c_{N_{c}-2} & \vdots & \vdots & \ddots & c_{1} & c_{0} \\
& & c_{N_{c}-1} & c_{N_{c}-2} & \ddots & \vdots & \vdots \\
& & & & & c_{N_{c}-1} & c_{N_{c}-2}
\end{array}\right] .
$$

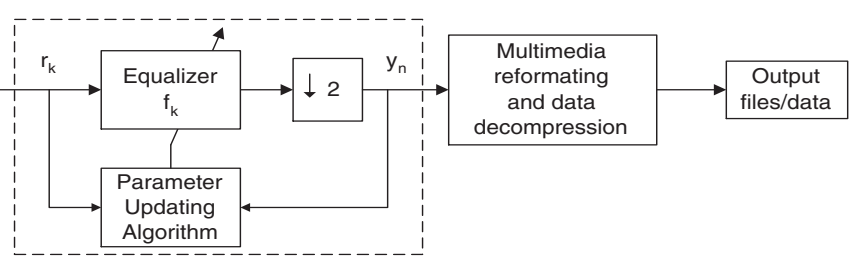

Fig. 2. Receiver block with a linear equalizer for $T / 2$ baseband communication system.

\section{A BLIND AdAPTIVE Algorithm}

Most of the blind adaptive equalizers update their coefficient vectors utilizing the stochastic gradient descent minimization method, which has also been used in this paper. The equalizer coefficients are updated according to the following algorithm:

$$
\mathbf{f}(n+1)=\mathbf{f}(n)+\mu \mathbf{r}(n) \psi_{\mathrm{cma}}\left(y_{n}\right),
$$

where $\mu$ is a small constant called the step-size, and $\psi_{\text {cma }}\left(y_{n}\right)$ is the CMA error function.

A complex-valued error function of CMA is described in [11] as:

$$
\psi_{\mathrm{cma}}\left(y_{n}\right)=y_{n}^{*}\left(\gamma-\left|y_{n}\right|^{2}\right),
$$

where $\gamma$ is a dispersion constant defined as

$$
\gamma=E\left[|x(n)|^{4}\right] / E\left[|x(n)|^{2}\right] .
$$

The objective that the equalizer minimizes the CM cost function (under a perfect blind equalization (PBE) condition [12]) is to remove all the linear distortion incurred by the channel such that the the output of the original source symbols are recovered for some system delay $\delta\left(0 \leq \delta \leq N_{x}-1\right)$ and phase shift $\theta(\theta \in[0,2 \pi])$. Hence, the filter output for perfect equalization becomes

$$
y_{n}=e^{j \theta} x_{n-\delta} .
$$

Recently, advanced studies of blind channel equalizers has moved towards the achievement of complexity reduction. Primarily, it is motivated by a requirement for low cost implementation of blind systems. One way to reduce complexity is by transforming the error function in eq. (4) into a sign function. It can be clearly shown, that the multiplication of every regressor element with the error function becomes a multiplication with +1 and -1 only. However, in order to preserve information lost in the quantization process a small perturbation random signal is added - known as dithering [14]. Thus the error function in eq. (4) for the complex-valued case is modified to:

$$
\begin{aligned}
\psi_{\text {dse-cma }}\left(y_{n}\right)= & \alpha \operatorname{sign}\left(\psi_{\text {cma }}^{r}\left(y_{n}\right)+\nu_{n}^{r}(\alpha)\right)+ \\
& j \alpha \operatorname{sign}\left(\psi_{\text {cma }}^{i}\left(y_{n}\right)+\nu_{n}^{i}(\alpha)\right),
\end{aligned}
$$

where $\alpha$ is a dither amplitude selected large enough to satisfy $\alpha \geq \max \left\{\left|\psi_{\mathrm{cma}}^{r}\left(y_{n}\right)\right|,\left|\psi_{\mathrm{cma}}^{i}\left(y_{n}\right)\right|\right\}, \nu_{n}^{r}(\alpha)$ and $\nu_{n}^{i}(\alpha)$ are realvalued i.i.d process sinusoidally distributed in $(-\alpha, \alpha)$ [13], where the superscripts $r$ and $i$ refer to the real and imaginary components, respectively. 
In order to guarantee that the sinusoidally-distributed dithered signed-error CMA (DSE-CMA) algorithm resembles the behavior of the CMA, the theorems of the average transient behavior DSE-CMA in [14] will be imposed, in which $\alpha$ is selected in accordance with the values of $\left\{\alpha_{\mathrm{c}}, \alpha_{\mathrm{ZF}}, \alpha_{\mathrm{OE}}\right\}$. These parameters are constellation and modulation dependent. The choice of $\alpha>\max \left\{\alpha_{\mathrm{c}}, \alpha_{\mathrm{zF}}\right\}$ guarantees the zero-forcing solutions of the equalizer to be in the vicinity of CMA minima, while selections of $\alpha>\alpha_{\text {OE }}$ ensure the mean trajectory within $\mathcal{F}_{\alpha}$. The convex hull $\mathcal{F}_{\alpha}$ is formed by the hyper planes $\mathcal{B}_{\alpha}:=\left\{\mathbf{f}:\left|\mathbf{r}^{H} \mathbf{f}\right|=\psi_{\text {cma }}^{-1}(\alpha)\right.$ for $\left.\mathbf{r} \in \mathcal{R}\right\}$ in the absence of channel noise, where $\mathcal{R}$ is the set of all possible received vector $\mathbf{r}$. The reader is referred to [15] for the complete design guidelines of $\alpha$.

\section{Modeling of Multimedia Data}

To enable the simulation of multimedia data over the multirate communication model, a process for converting multimedia signals from various data sources (such as, compressed audio/ image/ video files), which may be stored on a PC, has been developed. This has been integrated together with the DSE-CMA blind equalization scheme, to form the overall multimedia communication framework. Figure 3 depicts the general structure of the conversion process.

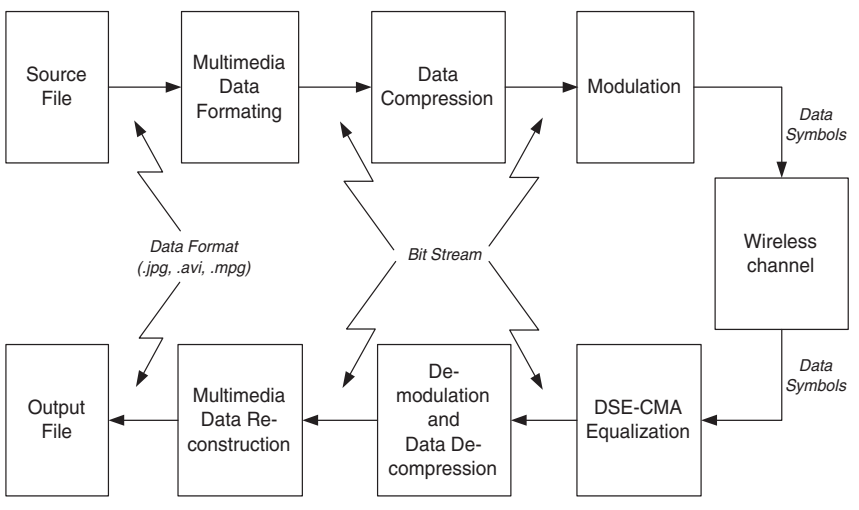

Fig. 3. Framework for wireless multimedia communication using DSE-CMA

The principal idea behind the multimedia data model developed is to first consider the source file (digitized version of the original multimedia signal with $\mathrm{N}$-bit resolution) as a vector array containing integer quantization levels. For digital audio, these correspond to sampled amplitude values, and for digital image and video coding they correspond directly to pixel values [16]. Before the multimedia data can be processed by the multirate communication model, the source data is converted into a matrix array containing $\mathrm{N}$ bit-words which correspond to the sample/ pixel levels. Thus, a bitstream representing the original multimedia data is created.

Our research framework includes the simulation of a 'data compression' block for system development along with investigating new adaptive compression algorithms. A channel encoder to convert the bitstream to the required baseband modulated data symbols $x_{n}$ as specified by the multirate model
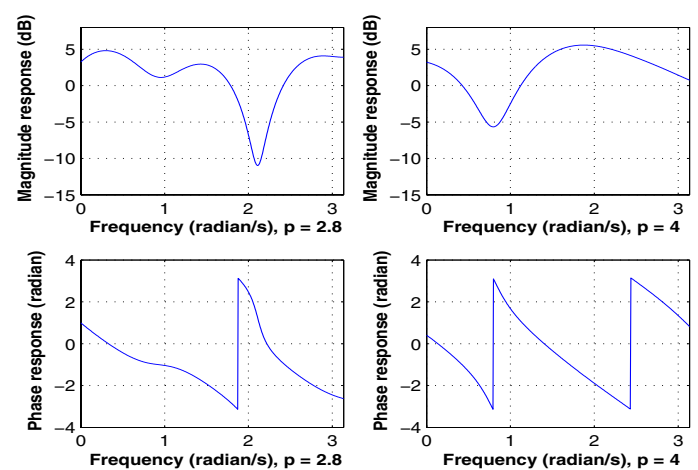

Fig. 4. Frequency responses of Channel A and Channel B with path loss exponent $(p=2.8)$ and $(p=4)$, respectively.

has been implemented. In the receiver path, the reverse is performed on the modified data symbols $y_{n}$ produced by the DSE-CMA equalizer. Whereby, the data symbols are first converted back to a bitstream by the channel decoder, and are subsequently reconstructed (via the de-compressor) to the original multimedia data format applied to the system.

\section{Simulation Results}

To evaluate the performance of the DSE-CMA equalization algorithm in the application of multimedia data, a test image is transmitted over various wireless communication channels using the geometrically-based hyperbolically distributed scatterers (GBHDS) channel model in [18]. Two kind of channels simulating urban environments are used, where Channel A is generated with a path loss exponent $(p=2.8)$ and Channel B is generated with a path loss exponent $(p=4)$. The frequency and phase responses of the channels are depicted in Figure 4. It can be clearly seen that both model frequency selective fading channels, with a very deep null produced by Channel A. These kind of channels obviously give rise to ISI as expected for multipath propagation in wireless environments, which deteriorate the received signal significantly.

A standard version of the $512 \times 512$ size gray scale Lena image with 8 bits/pixel resolution ${ }^{1}$ was used as the multimedia source data. However, due to practical computational limitations, the image was resized to $128 \times 128$ using 'bilinear' interpolation. The re-sized Lena image used for transmission is shown in Fig. 5. The overall communication framework was configured for QPSK baseband modulation. The remaining parameters of the fractionally-spaced equalizer were set as follows: $N_{f}=16$ and $\alpha=1$. The dither amplitude $\alpha$ was set to be large enough to guarantee the existence of the zeroforcing solutions of the equalizers.

Figure 6 shows the recovered image from the output of the channel with $p=2.8$, where there was no equalizer used at the input of the receiver. It was simulated with a $\mathrm{SNR}=50 \mathrm{~dB}$. As expected, this results an image which is severely distorted. Through visual inspection we can confirm that even in the

\footnotetext{
${ }^{1}$ located at http://www.dsp.ece.rice.edu/wakin/images/lena512
} 


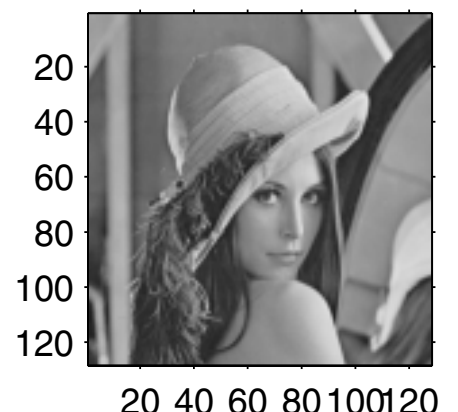

Fig. 5. Lena image source file (8-bit gray scale) after resizing to $128 \times 128$.

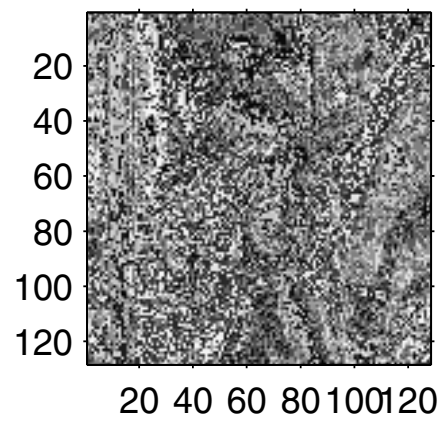

Fig. 6. Received Lena image file (8-bit gray scale) after passing it through the GBHDS channel $(p=2.8)$ without employing an equalizer, $\mathrm{SNR}=50 \mathrm{~dB}$.

absence of noise, channel-induced distortion leads to ISI and corruption of the original image.

In order to determine the effectiveness of the proposed DSE-CMA technique, the multirate model was configured and simulated for a SNR $=20 \mathrm{~dB}$ over a GBHDS channel $(p=2.8)$ using a blind adaptive equalizer based on two schemes. In the first scheme, we used the uniformly-distributed dithered signed-error CMA, and in the second case we employed our proposed sinusoidally-distributed dithered signederror CMA algorithm. Fig. 7 and Fig. 8 show the significant improvement obtained in using a sinusoidally-distributed DSE-CMA algorithm as opposed to the uniformly-distributed DSE-CMA method. This is due to the faster convergence rate of sinusoidally-distributed DSE-CMA as compared to uniformaly-distributed DSE-CMA [17].

Clearly, for different channel conditions the results obtained are similar. Fig. 9 and Fig. 10 show the equalized images recovered after employing the two algorithms, both simulated using the GBHDS channel model $(p=4)$. Under the condition when the thermal noise heavily corrupts the channel, Fig. 11 shows the degradation in user perceived quality of the received images. However, it can be seen that the equalizer is very

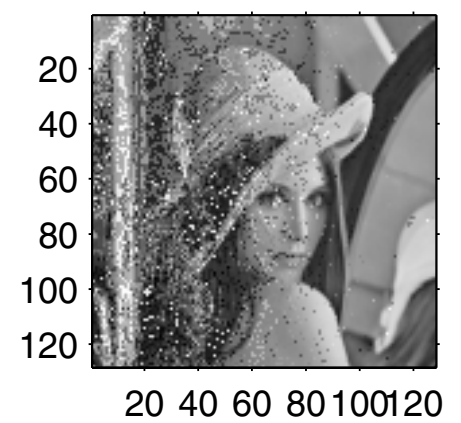

Fig. 7. Equalized Lena image file after passing it through the GBHDS channel $(p=2.8), \mathrm{SNR}=20 \mathrm{~dB}$ with blind adaptive equalization utilizing sinusoidally-distributed DSE-CMA.

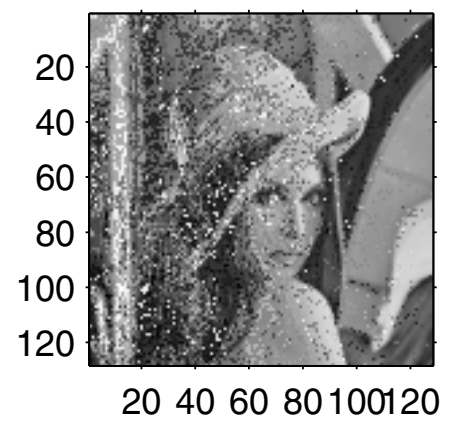

Fig. 8. Equalized Lena image file after passing it through the GBHDS channel $(p=2.8), \mathrm{SNR}=20 \mathrm{~dB}$ with blind adaptive equalization utilizing uniformly-distributed DSE-CMA.

efficient in removing the effect of ISI.

Further analysis was performed using an objective performance metric such as, the peak signal-to-noise ratio (PSNR), commonly used in image processing literature [3]. The PSNR performance curves of the received images, transmitted over various wireless GBHDS channels using the two blind equalization schemes, are plotted against different values of SNR. Each value of the PSNR for certain SNR values was averaged over 100 realizations. It can be seen in Fig. 12, that the sinusoidally-distributed DSE-CMA algorithm outperforms the uniformly-distributed DSE-CMA algorithm in both cases.

\section{CONCLUSION}

In this paper, we presented a multimedia communication framework to investigate the characteristics of DSE-CMA blind equalization in the transmission of compressed images over wireless communication channels. Simulation results (based on both user-perceived quality and the PSNR performance metric) showed that the recently-proposed DSE-CMA algorithm outperforms existing blind equalization techniques.

\section{REFERENCES}

[1] J. F. Huber, "Mobile next-generation networks," IEEE Multimedia, vol. 11, pp. 72-83, Jan. 2004. 


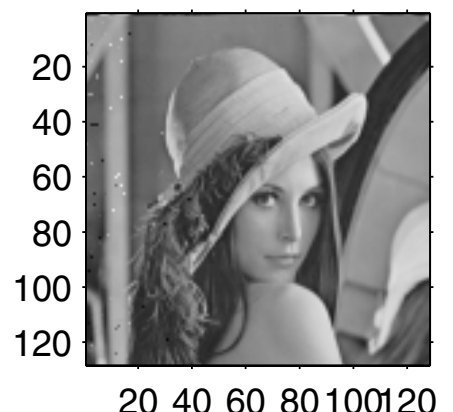

Fig. 9. Equalized Lena image file after passing it through the GBHDS channel $(p=4)$, SNR $=20 \mathrm{~dB}$ with blind adaptive equalization utilizing sinusoidally-distributed DSE-CMA.

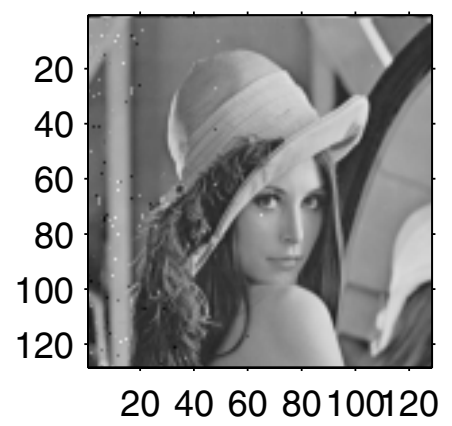

Fig. 10. Equalized Lena image file after passing it through the GBHDS channel $(p=4)$, SNR $=20 \mathrm{~dB}$ with blind adaptive equalization utilizing uniformly-distributed DSE-CMA.

[2] T. S. Rappaport, Wireless Communications Principles and Practice, 2ed Pearson Education Inc., Delhi, India, 2002.

[3] M. Ghanbari, Standard Codecs: Image Compression to Advanced Video Coding, The Institution of Electrical Engineers, IEE Telecommunications Series 49, Herts, UK, 2003.

[4] S. Catreux, V. Erceg, D. Gesbert, and R. W., Jr. Heath, "Adaptive modulation and MIMO coding for broadband wireless data networks," IEEE Commun. Magazine, v. 40, pp. 108-115, Jun 2002.

[5] C. N. Taylor and S. Dey, "Adaptive image compression for wireless multimedia communication," IEEE ICC, pp. 1925-1929, 2001.

[6] J. Yang, M. H. Lee, M. Jiang, and J. Y. Park, "Robust wireless image transmission based on turbo-coded OFDM," IEEE Trans. Consumer Elec., v. 48 , no. 3, pp. 724-730, Aug. 2002.

[7] J. Hagenauer and T. Stockhammer, "Channel coding and transmission aspects for wireless multimedia," Proc. IEEE, v. 3, pp. 1764-1777, Oct. 1999.

[8] Q. Liu, S. Zhou, and G. B. Giannakis, "Cross-Layer combining of adaptive Modulation and coding with truncated ARQ over wireless links," IEEE Trans. Wireless Commun., v. 87, pp. 1746-1755, Sep. 2004.

[9] T. Athanasiadis, K. H. Lin, and Z. M. Hussain, "Space-time OFDM with Adaptive Beamforming for Wireless Multimedia Applications," Proc. IEEE International Conference on Information Technology and Applications (ICITA 2005), Sydney, Jul. 2004.

[10] J.G. Proakis, Digital Communications, 3ed, McGraw Hill International, New York, USA, 1995.

[11] D. Godard, "Self-recovering equalization and carrier tracking in two dimensional data communication systems," IEEE Trans. Commun., v. 28, pp. 1867-1875, 1980.

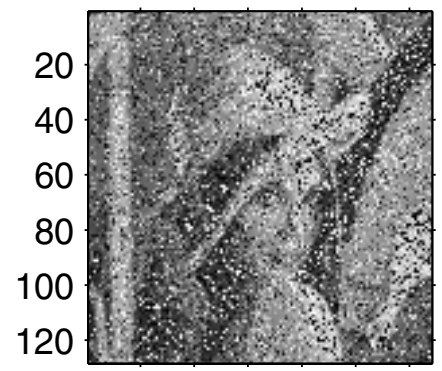

20406080100120

Fig. 11. Equalized Lena image file after passing it through the GBHDS channel $(p=4)$, SNR $=5 \mathrm{~dB}$ with blind adaptive equalization utilizing sinusoidally-distributed DSE-CMA.
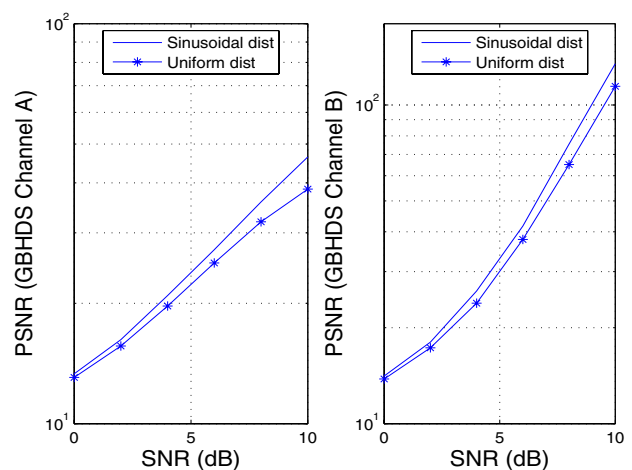

Fig. 12. Peak signal-to-noise ratio (PSNR) of the received Lena image after equalization for different values of SNR.

[12] C.R. Johnson, Jr., P. Schniter, T.J. Endres, J.D. Behm, D.R. Brown, and R.A. Casas, "Blind equalization using the constant modulus criterion: A review," Proc. IEEE, v. 86, no. 10, pp. 1927-1950, Oct. 1998.

[13] J. Jusak and Z. M. Hussain, "Evaluation of a sinusoidally-distributed dithering for signed-error constant modulus algorithm: performance comparison with uniformly-distributed dithering," Proc. The 3rd Workshop on the Internet, Telecommunications and Signal Processing (WITSP), Adelaide, Australia, Dec. 2004.

[14] P. Schniter and R. J. Johnson, Jr., "Dithered signed-error CMA: the complex-valued case," Proc. 32nd Asilomar Conf. on Signals, Systems, and Computers, pp. 1143-1147, 1998.

[15] P. Schniter and R.J. Johnson, Jr., "Dithered signed-error: robust, computationally efficient blind adaptive equalization," IEEE Trans. Signal Processing, v. 47, no. 6, pp. 1592-1603, 1999.

[16] M. Kr. Mandal, Multimedia Signals and Systems, Kluwer Academic, Massachusetts, USA, 2003.

[17] J. Jusak and Z. M. Hussain, "Performance of sinusoidally-distributed dithering for signed-error constant modulus algorithm," Proc. IEEE International Conference on Information Technology and Applications (ICITA 2005), Sydney, Australia, Jul. 2005.

[18] S. S. Mahmoud, Z. M. Hussain and P. O'Shea "A space-time model for mobile radio channel with hyperbolically distributed scatterers ," IEEE Antennas and Wireless Propagation Letters, v. 1, no. 1, pp. 211-214, 2002 . 